\title{
Article \\ Comparative Evaluation of Quality and Metabolite Profiles in Meju Using Starter Cultures of Bacillus velezensis and Aspergillus oryzae
}

\author{
Na-Young Gil, Ye-Ji Jang, Hee-Min Gwon, Woo-Soo Jeong, Soo-Hwan Yeo and So-Young Kim *D
}

Citation: Gil, N.-Y.; Jang, Y.-J.; Gwon, H.-M.; Jeong, W.-S.; Yeo, S.-H.; Kim, S.-Y. Comparative Evaluation of Quality and Metabolite Profiles in Meju Using Starter Cultures of Bacillus velezensis and Aspergillus oryzae. Foods 2022, 11, 68. https:// doi.org/10.3390/foods11010068

Academic Editor: Luciana De Vero

Received: 30 October 2021

Accepted: 24 December 2021

Published: 28 December 2021

Publisher's Note: MDPI stays neutral with regard to jurisdictional claims in published maps and institutional affiliations.

Copyright: (C) 2021 by the authors. Licensee MDPI, Basel, Switzerland. This article is an open access article distributed under the terms and conditions of the Creative Commons Attribution (CC BY) license (https:// creativecommons.org/licenses/by/ $4.0 /$ )
Fermented and Processed Food Science Division, Department of Agrofood Resources, National Institute of Agricultural Science, RDA, 166, Nongsaengmyeong-ro, Iseo-myeon, Wanju 55365, Korea; 01077510220@hanmail.net (N.-Y.G.); rowlsla94@korea.kr (Y.-J.J.); vitamin89@korea.kr (H.-M.G.); wjddntnek@korea.kr (W.-S.J.); yeobio@korea.kr (S.-H.Y.)

* Correspondence: foodksy@korea.kr; Tel.: +82-63-238-3610; Fax: +82-63-238-3843
Abstract: The production of good Meju soybean paste primarily depends on the selection of raw materials and fermenting microorganisms, which together influence its characteristic metabolites, taste, and aroma. In this study, we analyzed the relationship between properties and metabolites in Meju samples fermented by Aspergillus oryzae alone or with Bacillus velezensis. We developed fast-stable processing techniques to obtain Meju from A. oryzae and B. velezensis using the inoculation method, thereby ensuring safety in the production of soybean paste. The amino-type nitrogen content increased from an initial 180-228 mg\% to a final 226-776 mg\% during fermentation and was higher in Meju inoculated separately with the fungi and bacteria (C group) than in Meju co-inoculated with both the starters concurrently (D group). The levels of metabolites such as glucose, myo-inositol, glycerol, and fatty acids (palmitic, stearic, oleic, and linoleic acids) in Meju fermented by A. oryzae with $B$. velezensis were higher than those in Meju fermented by A. oryzae alone. Fungal growth was affected by the inoculated bacteria, which often occurs during the fermentation of co-inoculated Meju.

Keywords: Aspergillus oryzae; Bacillus velezensis; Meju; metabolites

\section{Introduction}

Soybeans are an important raw material commonly used in food manufacturing in Asian countries, including Korea, China, Indonesia, and Japan, owing to their high content of protein and oil (approximately $40 \%$ and $20 \%$ of their dry weight, respectively) [1]. Many studies have been conducted on the salubrious effects of soybeans and their products, such as soybean paste and sauce, particularly the anti-cancer effects and amelioration of cardiovascular and other chronic diseases, indicating that this is an important food ingredient $[2,3]$.

Meju, an unsalted representataive of fermented soybean food in Korea, is used in the production of soybean paste (Doenjang), soy sauce (Ganjang), and red pepper paste (Gochujang). Meju is classified into two types, traditional and modified, according to the manufacturing method. Traditional Meju is naturally fermented by mold or bacteria while the steamed beans dry, whereas modified Meju is koji made by inoculating the microbial starters into raw starch materials, such as rice, wheat, or barley [4]. Various fungi (including the genera Aspergillus, Mucor, and Rhizopus) and bacteria (including the genera Bacillus and Staphylococcus) have been found in Meju during fermentation [5,6]. Owing to their capacity to produce various enzymes, these microorganisms can degrade proteins and carbohydrates into small molecule compounds, such as amino acids and fatty acids [7]. Of these microorganisms, Aspergillus oryzae has been widely used in the food industry as a starter to manufacturing modified $M e j u$, but many types of bacilli and fungi have been found in Meju. However, biogenic amines, such as tyramine, histamine, and putrescine, 
or biohazards, such as Bacillus cereus or Aspergillus flavus, can also occasionally occur [8]. Therefore, many studies have attempted to use a bacilli starter to control these hazards in the production of traditional fermented soybean products. Moreoever, a single starter, such as bacilli, that reduces the relevant harmful factors for these products to solve these problems has been developed. However, a bacilli starter inhibits useful fungi, such as A. oryzae. Accordingly, a solution to co-grow both bacilli and mold starters should be developed.

Bacillus sp. produces antifungal and antimicrobial agents, but research using it as a starter for fermented soy products is lacking [9]. The Ministry of Food and Drug Safety limits edible bacterial to only six species of Bacillus: B. amyloliquefaciens, B. velezensis, $B$. coagulans, B. natto, B. polyfermenticus, and B. subtilis [10]. B. velezensis was proposed as a subspecies strain of $B$. amyloliquefaciens subsp. plantarum but has recently been recognized by physical analysis as a synonym for B. methylotrophicus [11,12]. B. velezensis has been reported to have salt tolerance and high enzymatic activity [13]. Studies on B. velezensis have isolated and characterized strains with antifungal activity from Korean traditional soy sauce for application as starters $[14,15]$.

The aim of study was to compare the fermentative changes associated with using B. velezensis with $A$. oryzae as starters in Meju fermentation and to investigate the changes in quality characteristics, metabolites, and microbial community using gas chromatographymass spectrometry (GC-MS) and next-generation sequencing analysis. Furthermore, $B$. velezensis was evaluated by establishing a manufacturing process that considers antagonism with A. oryzae.

\section{Materials and Methods}

\subsection{Manufacturing of the Modified Meju}

In this study, we prepared Meju by using the modified method with bacilli and fungi starters to ferment soybeans. Based on a previous study, B. amyloliquefaciens NY12-2 (reclassified as B. velezensis NY12-2, Accession: NZ_CP033576), which is used to ferment Meju, was selected as a bacterium that inhibits B. cereus [16]. A. oryzae KCTC 46471 was purchased from the Korean Collection for Type Cultures (KCTC, Daejeon, Korea). The experimental groups were divided into four Meju: control without a starter (Group (A)), A. oryzae single inoculation (Group (B)), B. velezensis and A. oryzae individual inoculation in each other chamber Group $(C)$, and B. velezensis and A. oryzae co-inoculation in a chamber Group (D).

After cleaning and soaking $10 \mathrm{~kg}$ of soybeans (Daewon cultivar (Andong, Korea)) in water for $15 \mathrm{~h}$, the soybeans were steamed at $121^{\circ} \mathrm{C}$ for $60 \mathrm{~min}$. The cooled steamed beans were inoculated with B. velezensis NY12-2 $\left(1 \times 10^{8} \mathrm{CFU} / \mathrm{mL}\right.$ in saline, $\left.1 \%\right)$ and A. oryzae KCTC $46471\left(1 \times 10^{7}\right.$ spores $/ \mathrm{mL}$ in saline, $\left.1 \%\right)$ and then incubated at $30^{\circ} \mathrm{C}$ for 1 day.

\subsection{Physicochemical Properties Analysis}

The moisture content of Meju was analyzed at $105^{\circ} \mathrm{C}$ by drying according to the Association of Official Analytical Chemists method [17]. Two grams of each sample were dried in a $105^{\circ} \mathrm{C}$ dry oven (MOV-112, Sanyo Co., Ltd., Osaka, Japan) until a constant weight was reached, which was expressed as a percentage.

For preparation for $\mathrm{pH}$ measurement, $50 \mathrm{~g}$ of $\mathrm{Meju}$ samples were diluted five times with $200 \mathrm{~mL}$ of distilled water, homogenized using a PT-MR 2100 homogenizer (Kinematica, Lucerne, Switzerland) for $1 \mathrm{~min}$, and filtered through Whatman No. 2 filter paper. $\mathrm{pH}$ was measured using an Orion star a211 pH meter (Thermo Fisher Scientific, Beverly, MA, USA). The acidity was calculated by adding $0.5 \%$ phenolphthalein as an indicator to $10 \mathrm{~mL}$ of the sample solution and titrating with $0.1 \mathrm{~N} \mathrm{NaOH}$ until it became reddish. The amino-type nitrogen content was measured via the formalin titration method. The $\mathrm{pH}$ of the sample solution was adjusted to 8.4 by adding $0.1 \mathrm{~N} \mathrm{NaOH}$; then, $10 \mathrm{~mL}$ of neutral formalin was added to the solution, which was then titrated with $\mathrm{NaOH}(0.1 \mathrm{~N})$ to reach $\mathrm{pH}$ 8.3-8.4. 


\subsection{Total Viable Counts and MiSeq Metagenomic Sequencing}

To count the total viable bacteria and fungi in each Meju sample, $1 \mathrm{~g}$ of sample was mixed in $9 \mathrm{~mL}$ of physiological saline $(0.85 \%(w / v) \mathrm{NaCl})$ to accomplish a ten-fold dilution and homogenized using a Vortex-Genie 2 (Scientific Industries, Inc., Bohemia, NY, USA). The homogenate was serially diluted with saline solution. Diluted samples $(0.05 \mathrm{~mL})$ were inoculated on tryptic soy agar or potato dextrose agar plates and incubated for 24 or $48 \mathrm{~h}$ at $30{ }^{\circ} \mathrm{C}$, respectively. Total counts were calculated as typical colonies identified on each agar plate, considering dilutions presumed to be bacilli (with dehydrated big colony) or mold (with mycelium; for comparison, yeast forms a white opaque colony).

Metagenome sequencing was performed to identify microbial communities in different Meju environments. DNA was extracted using a PowerSoil ${ }^{\circ D N A}$ Isolation Kit (Cat. No. 12888, MO BIO, Carlsbad, CA, USA) according to the manufacturer's protocol. Each sequenced sample was prepared according to the Illumina Sequencing Library protocols. DNA quantification and quality were analyzed using Quant-iT ${ }^{\mathrm{TM}}$ PicoGreen and Nanodrop ND-1000 (Thermo Fisher Scientific, Beverly, MA, USA). The 16S rRNA genes were amplified using V3-V4 region (forward: $5^{\prime}$-TCGTCGGCAGCGTCAGATGTGTATAAGAGACAGCCT ACGGGNGGCWGCAG-3' ${ }^{\prime}$; reverse: $5^{\prime}$-GTCTCGTGGGCTCGGAGATGTGTATAAGAGAC AGGACTACHVGGGTATCTAATCC-3') and ITS3-ITS4 (forward: $5^{\prime}$-TCGTCGGCAGCGTC AGATGTGTATAAGAGACAGGCATCGATGAAGAACGCAGC-3' ; reverse: $5^{\prime}$-GTCTCGTG GGCTCGGAGATGTGTATAAGAGACAGTCCTCCGCTTATTGATATGC-3') primers. Input gDNA was amplified with 16S rRNA V3-V4 or 5.8-28S rRNA ITS3-ITS4 primer sets, and multiplexing indices and Illumina sequencing adapters were added by subsequent limited-cycle amplification. The final products were normalized and pooled using PicoGreen, and the library sizes were verified using the TapeStation DNA D1000 screen tape assay (Agilent, Santa Clara, CA, USA). The libraries were then sequenced using MiSeq ${ }^{\mathrm{TM}}$ (Illumina, San Diego, CA, USA) by Macrogen (Seoul, Korea).

\subsection{Microbial Community Metagenome Using PICRUSt Based on $16 S$ rRNA Sequencing Data}

PICRUSt analysis was used to predict the microbial community metagenome using the Greengene database based on taxonomic abundance, as detailed by Langille et al. [18].

All predicted gene families were classified using the Kyoto Encyclopedia of Genes and Genomes (KEGG) pathway database (https:/ / www.genome.jp/kegg/ kegg2.html, accessed on 19 May 2020). The functions of genes and their relative abundances in the predicted metagenome were compared among the Meju samples and visualized in a heatmap using the omics function in XLStat (XLStat).

\subsection{Volatile Analysis by GC-MS}

The volatile compounds were measured by adding $1 \mathrm{~g}$ of sample, $1 \mathrm{~g}$ of $\mathrm{NaCl}$, and $4 \mathrm{~mL}$ of distilled water (including internal standard) into a $20 \mathrm{~mL}$ glass vial, heating at $40^{\circ} \mathrm{C}$, stirring for $15 \mathrm{~min}$, and adsorbing for $10 \mathrm{~min}$ with solid-phase microextraction (SPME). The volatile compounds were analyzed using a GC-2010 Plus gas chromatograph (Shimadzu, Kyoto, Japan) equipped with a DB-WAX column $(30 \mathrm{~mm} \times 0.25 \mathrm{~mm}$ i.d. $\times 0.25 \mu \mathrm{m}$ film; $\mathrm{J} \& \mathrm{~W}$ Scientific, Folsom, CA, USA). The flow rate of the helium carrier gas was maintained at $1 \mathrm{~mL} / \mathrm{min}$, and the injector temperature was set to $250^{\circ} \mathrm{C}$. The initial oven temperature was maintained at $40{ }^{\circ} \mathrm{C}$ for $3 \mathrm{~min}$, raised to $90{ }^{\circ} \mathrm{C}$ at $5^{\circ} \mathrm{C} / \mathrm{min}$, raised again to $230{ }^{\circ} \mathrm{C}$ at $19^{\circ} \mathrm{C} / \mathrm{min}$, and then maintained for $5 \mathrm{~min}$. The mass spectra were analyzed using a GC-MS-TQ 8030 (Shimadzu) and monitored in the Q3 scan mode. The temperatures of the ion interface and source were set to $280{ }^{\circ} \mathrm{C}$ and $230^{\circ} \mathrm{C}$, respectively. A detector voltage of $0.1 \mathrm{kV}$ was used.

\subsection{Metabolite Analysis}

For metabolite analysis, Meju at a concentration of $0.02 \mathrm{~g} / 500 \mu \mathrm{L}$ in $80 \%$ methanol was extracted using a blender. The extract was centrifuged for $10 \mathrm{~min}$ at $16,127 \times g$ and $25^{\circ} \mathrm{C}$, and then $10 \mu \mathrm{L}$ of the obtained supernatant was dried using a speed vac concen- 
trator (Labconco Co., Kansas City, MO, USA). Derivatization was performed at $37{ }^{\circ} \mathrm{C}$ for $90 \mathrm{~min}$ after adding $70 \mu \mathrm{L}$ hydroxymethoxy amine. Subsequently, $70 \mu \mathrm{L}$ of $N, \mathrm{O}_{-}$ bis(trimethylsilyl)trifluoroacetamide was added, and the mixture was incubated to $70{ }^{\circ} \mathrm{C}$ for $30 \mathrm{~min}$. The derivatized reactant was centrifuged for $10 \mathrm{~min}$ at $16,127 \times g$ to obtain the supernatant. Relative quantification of the metabolites was performed using GC-MS.

Metabolites of the Meju samples were analyzed using a GC-2010 Plus (Shimadzu, Tokyo, Japan) equipped with a DB-WAX column $(30 \mathrm{~m} \times 0.25 \mathrm{~mm}$ i.d. $\times 0.25 \mu \mathrm{m}$; J\&W Scientific, Santa Clara, CA, USA). The flow rate of the helium carrier gas was maintained at $1 \mathrm{~mL} / \mathrm{min}$, and the injector temperature was set at $200^{\circ} \mathrm{C}$. The oven temperature was held at $80{ }^{\circ} \mathrm{C}$ for $2 \mathrm{~min}$ and then raised at $10{ }^{\circ} \mathrm{C} / \mathrm{min}$ to $320^{\circ} \mathrm{C}$, where it was maintained for $6 \mathrm{~min}$. MS analysis was performed under the same conditions.

MS data analysis was conducted using Pro software (Spectralworks Ltd., Runcorn, UK). Metabolites were calculated using n-alkanes and confirmed by comparing the mass spectra and retention indices with the Wiley and National Institute of Standards and Technology mass spectral databases. The resulting data were input to SIMCA-P+ version 12.0 (Umetrics, Umea, Sweden) for collection, alignment, and normalization. Metabolite profiles were displayed as heatmaps using XLStat (XLStat, New York, NY, USA).

\subsection{Statistical Analysis}

All experiments were performed in triplicate, and the analyzed data are presented as means \pm standard deviation (SD). MS data were visualized by principal component analysis (PCA) and partial least squares discriminant analysis (PLS-DA). $p$-values were determined using SAS software (SAS Institute, Cary, NC, USA), and those below the threshold of $p=0.05$ were considered statistically significant. PCA and heatmap analysis were performed using XLStat for Microsoft Excel (Microsoft Corp., Redmond, WA, USA). Significant differences of all data, including physicochemical components, viable microbial counts, and metabolites, were tested by one-way analysis of variance (ANOVA) with Duncan's multiple range test using SPSS version 17.0 (SPSS Inc., Chicago, IL, USA).

\section{Results and Discussion}

In our study, we aimed to develop a fast-stable processing technique to obtain Meju from $A$. oryzae and $B$. velezensis by the inoculation method.

\subsection{Physicochemical Properties of Modified Meju}

We fermented Meju for $24 \mathrm{~h}$ at $30{ }^{\circ} \mathrm{C}$ using starters of $A$. oryzae with or without B. velezensis. The qualitative properties and viable microbial counts are presented in Table 1. The initial moisture and $\mathrm{pH}$ of all Meju samples were 58.18-60.12\% and 6.46-6.62, respectively, which decreased to $57.66 \%-59.96 \%$ and $6.28-6.57$, respectively, after 1 day of fermentation. There was no significant difference in the moisture content among the Meju samples. Group B had the lowest pH (6.28) after 24 h, but no notable difference was observed between the experimental groups and the fermentation period.

The acidity $(0.95 \%)$ in sample $C$ dramatically increased by the end of the fermentation process. The acidity of sample A did not change during the fermentation period, but that of sample B increased significantly more than that of sample D as the fermentation progressed $(p<0.05)$.

Here, we doubted the survival of $A$. oryzae in sample D. There are no published reports of $B$. velezensis inhibiting $A$. oryzae, which is non-pathogenic. However, Bacillus sp. has antifungal effects against molds, such as Aspergillus niger and Rhizopus spp. [19]. Furthermore, Choi et al. reported differences in fermentative activity and acid production depending on the fungal strain used [20]. In their study, A. oryzae and Rhizopus oligosporus showed titratable activity at $1.35-1.40 \%$ and $1.60-1.75 \%$, respectively, which was higher than that in commercial Meju without fungi (0.88\%). 
Table 1. Changes in moisture, $\mathrm{pH}$, titratable acidity (\%), amino-type nitrogen ( $\mathrm{mg} \%)$, and viable microbial counts in Meju samples.

\begin{tabular}{|c|c|c|c|c|c|c|c|}
\hline \multirow{2}{*}{ Groups } & \multirow{2}{*}{$\begin{array}{l}\text { Times } \\
\text { (h) }\end{array}$} & \multicolumn{4}{|c|}{$\begin{array}{l}\text { Quality Properties } \\
(\%, \text { Except for } \mathrm{pH})\end{array}$} & \multicolumn{2}{|c|}{$\begin{array}{c}\text { Viable Microbial Counts } \\
(\log \text { CFU/g) }\end{array}$} \\
\hline & & Moisture & $\mathrm{pH}$ & $\begin{array}{l}\text { Titratable } \\
\text { Acidity }\end{array}$ & $\begin{array}{l}\text { Amino-Type } \\
\text { Nitrogen }\end{array}$ & $\begin{array}{l}\text { Aerobic } \\
\text { Bacteria }\end{array}$ & Mold \\
\hline \multirow{2}{*}{ A } & 0 & $60.12 \pm 1.90^{a}$ & $6.52 \pm 0.01^{\mathrm{e}}$ & $0.38 \pm 0.00$ ef & $180.0 \pm 11.1^{\mathrm{d}}$ & $2.30 \pm 0.00 \mathrm{bc}$ & $0.00 \pm 0.00^{d}$ \\
\hline & 24 & $59.21 \pm 2.97^{\mathrm{a}}$ & $6.57 \pm 0.00^{b}$ & $0.42 \pm 0.00^{\mathrm{d}}$ & $226.0 \pm 11.0 \mathrm{bc}$ & $7.93 \pm 0.06^{\mathrm{a}}$ & $0.00 \pm 0.00^{d}$ \\
\hline \multirow{2}{*}{ B } & 0 & $60.03 \pm 2.31^{a}$ & $6.62 \pm 0.01^{\mathrm{a}}$ & $0.32 \pm 0.01 \mathrm{~g}$ & $260.0 \pm 11.1^{\mathrm{b}}$ & $0.00 \pm 0.00^{c}$ & $5.46 \pm 0.17^{b}$ \\
\hline & 24 & $59.96 \pm 1.66^{\mathrm{a}}$ & $6.28 \pm 0.00^{\mathrm{h}}$ & $0.78 \pm 0.00^{b}$ & $776.0 \pm 11.1^{\mathrm{a}}$ & $8.41 \pm 0.26^{\mathrm{a}}$ & $6.45 \pm 0.16^{a}$ \\
\hline \multirow{2}{*}{$\mathrm{C}$} & 0 & $58.18 \pm 0.71^{a}$ & $6.54 \pm 0.00^{\mathrm{d}}$ & $0.37 \pm 0.00^{f}$ & $227.0 \pm 11.1^{b c}$ & $2.15 \pm 3.04^{b c}$ & $4.67 \pm 0.02^{c}$ \\
\hline & 24 & $58.15 \pm 1.35^{\mathrm{a}}$ & $6.32 \pm 0.00^{g}$ & $0.95 \pm 0.00^{\mathrm{a}}$ & $776.0 \pm 11.1^{\mathrm{a}}$ & $9.40 \pm 0.02^{\mathrm{a}}$ & $6.44 \pm 0.04^{\mathrm{a}}$ \\
\hline \multirow{2}{*}{$\mathrm{D}$} & 0 & $58.66 \pm 0.32^{a}$ & $6.46 \pm 0.00^{f}$ & $0.40 \pm 0.01^{\mathrm{e}}$ & $196.0 \pm 11.1^{\mathrm{cd}}$ & $5.77 \pm 0.38^{\mathrm{ab}}$ & $5.62 \pm 0.09^{b}$ \\
\hline & 24 & $57.66 \pm 0.29^{a}$ & $6.55 \pm 0.00^{c}$ & $0.68 \pm 0.01^{c}$ & $172.0 \pm 0.0^{\mathrm{d}}$ & $9.82 \pm 0.05^{\mathrm{a}}$ & $4.31 \pm 0.08^{c}$ \\
\hline
\end{tabular}

The experimental groups were divided into four Meju: control without a starter (A), A. oryzae KCTC 46471 single inoculation Group (B), B. velezensis and A. oryzae individual inoculation Group (C), and B. velezensis and A. oryzae co-inoculation Group (D) between pre- and post-fermentation for $0-24 \mathrm{~h}$. Values are presented as means \pm standard deviation (number of repetitions $=3$ ). Superscripts in the same column not sharing a common superscript are significantly different at $p<0.05$ by Duncan's multiple range test.

In addition, the content of amino-type nitrogen is the key component in soybean fermentation, and the content can increase from an initial $180-228 \mathrm{mg} \%$ to a final $226-776 \mathrm{mg} \%$ during fermentation. Samples B and C had relatively high final content $(776 \mathrm{mg} \%)$, whereas that of sample $\mathrm{D}$ did not increase during the same period. The high amino-type nitrogen content in fungi-bacteria-fermented Meju can be correlated to the viability of $A$. oryzae by the antifungal effect of $B$. velezensis. B. velezensis inhibits the growth of pathogenic fungi, such as A. flavus, Fusarium graminearum, and A. oryzae, and reduces their ability to produce the mycotoxins aflatoxin and ochratoxin [21].

\subsection{Effect of Starter Inoculation Method on Changes in Bacterial and Fungal Communities}

The total viable bacterial cell count in all experimental groups increased after fermentation, with Group $C$ having the highest count $(9.40 \pm 0.02 \mathrm{cfu} / \mathrm{g}$; Table 1$)$. Mold was not found even after fermentation for $24 \mathrm{~h}$ in Group A, but the mold count increased in Groups $\mathrm{B}$ and $\mathrm{C}$. In the case of Group D, the total decreased as fermentation progressed. B. cereus was not detected in any experimental group.

In this study, the diversity of bacteria and fungi did not vary during the improved Meju fermentation using starters. The sequencing reads for the bacteria and fungi were classified at the genus and species levels to compare changes in the microbial communities among the four groups after 1 day of fermentation. The evaluation of the bacterial community at the genus level indicated that the genera Bacillus and Aerosakkonema predominated; the relative abundances ratio in all samples was 3.6-94.0:15.5-86.3, respectively (Figure 1a). At the species level, bacterial community analysis showed that the relative abundances ratio of B. velezensis and Aerosakkonema funiforme before and after fermentation was notably different depending on the method used to inoculate starters. In Group B, there was no significant difference in bacterial composition before and after fermentation. A. funiforme initially predominated (86.3-86.8\%), but after $24 \mathrm{~h}$, B. velezensis was detected at a level of $4 \%$ without starter inoculation. In contrast, $B$. velezensis in samples $C$ and $D$ increased from $60.7 \%$ to $94.0 \%$ and from $49.0 \%$ to $86.3 \%$, respectively. In addition, A. funiforme in samples C and D decreased from $37.5 \%$ to $5.9 \%$ and from $43.4 \%$ to $13.5 \%$, respectively. Immediately after inoculation, other bacteria found in samples $C$ and $D$ showed the relative abundances ratio of $1.5-7.6 \%$. After 1 day, the relative abundance ratio of the other bacteria decreased to $0.1-0.2 \%$ owing to the growth of bacillus spp (except for Group B, which showed a low relative abundance ratio of bacilli). A. funiforme, which is included in the phylum Cyanobacteria, was first reported to be isolated from mesotrophic reservoirs by 
Thu et al. [22]. To the best of our knowledge, this is the first report of A. funiforme being predominant in Meju.

(a)

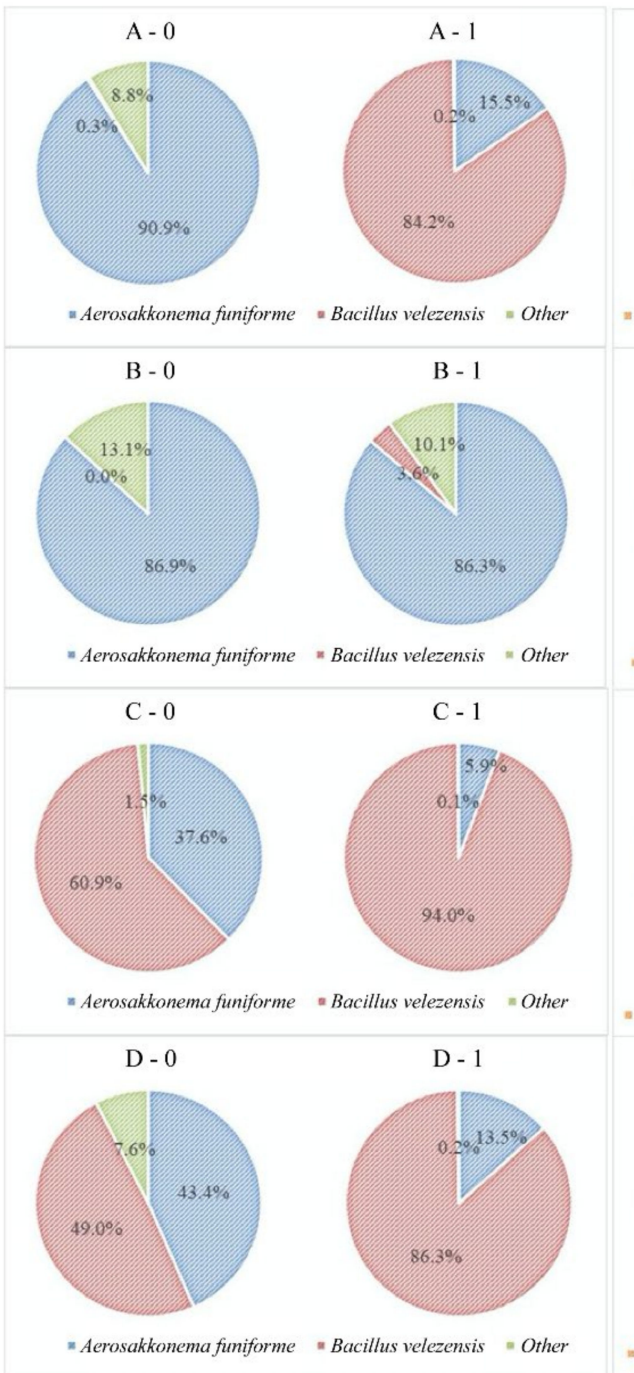

(b)
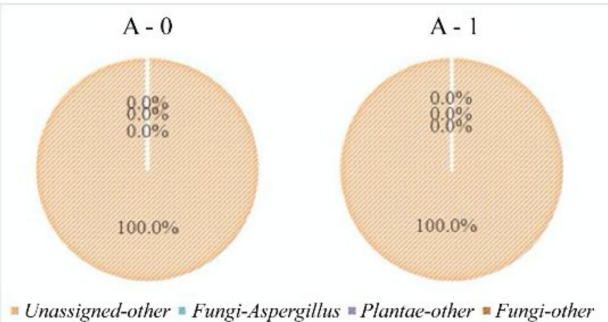

Unassigned-other $=$ Fungi-Aspergillus $\|$ Plantae-other $=$ Fungi-other

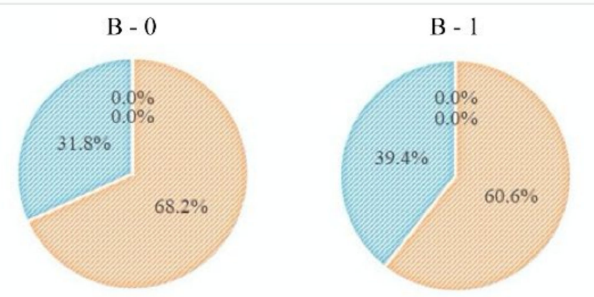

- Unassigned-other $=$ Fungi-Aspergillus $=$ Plantae-other $=$ Fungi-other C -0 $\mathrm{C}-1$

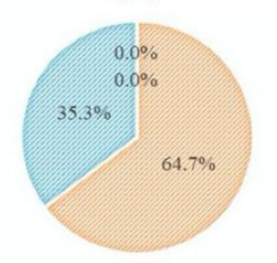

" Unassigned-other $=$ Fungi-Aspergillus $=$ Plantae-other "Fungi-other

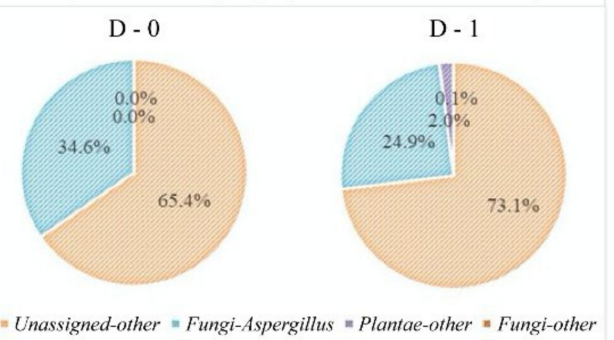

Figure 1. Taxonomic classification at the species level of the (a) bacterial $16 \mathrm{~S}$ rRNA gene and (b) fungal ITS gene reveals changes in bacterial and fungal communities during one-day fermentation. The experimental groups were inoculated as follows: A, control without starter; B, Aspergillus oryzae single inoculation group; C, Bacillus velezensis and A. oryzae individual inoculation group; and D, $B$. velezensis and $A$. oryzae co-inoculation group.

Phylum-level analysis of the fungal community revealed that unassigned phyla and Aspergillus were the major eukaryotes in the Meju samples (Figure 1b), which agrees with the results of a previous study [23]. In all inoculated Meju samples, Aspergillus was predominant with a relative abundance of $24.9-96.8 \%$, and the relative abundances of unassigned eukaryotes were also high at $3.2-73.1 \%$. However, their relative abundances before and after fermentation varied depending on the starter inoculation method. These results indicate that the growth of $A$. oryzae is closely related to an increase in fungal abundance. However, the relative abundance ratio of Aspergillus in sample B was higher than that in sample $\mathrm{D}$ because of the antifungal effect of $B$. velezensis [21]. These results suggest that the growth of fungi in the presence of the co-inoculated bacteria often reduces during fermentation. When Meju is manufactured using combination starters, we should consider their antagonism effect and suitable inoculation method. 


\subsection{Volatile Components}

The flavor characteristics of the fermented Meju samples were analyzed using GC-MS and headspace SPME. The total of 35 volatile compounds identified in all Meju samples included 10 esters, 13 alcohols, 1 acid, 2 aldehydes, 6 ketones, 2 pyrazines, and 1 other species. The main flavor volatiles, ethyl acetate, methylene chloride, and ethanol, were detected at a high abundance at $0 \mathrm{~h}$ of fermentation. The content of ethyl acetate and ethanol increased, whereas that of methylene chloride decreased after $24 \mathrm{~h}$ of fermentation. Ethanol is produced by the reduction in carbonyl compounds of aldehydes, which correspond to soybean protein or fat metabolites, but most have been reported as metabolites converting glucose to ethanol during soybean fermentation.

The 3-methyl-1-butanol content was relatively high in Groups B and C. This component produces soybean odor as a degradation product of leucine, which decreases upon heating. 2,3-Butanediol was produced during fermentation and was an intermediate of 3-hydroxy-2-butanone (acetoin) produced by polymerization with the thiamine pyrophosphate catalyst of ethanol. The proportions of other flavor components were generally less than $1 \%$, with no significant difference among the Meju samples. This is because the flavor component of $M e j u$ is produced by the Maillard reaction during the fermentation of soy protein, fat, and carbohydrates and various enzymatic metabolic reactions by microorganisms and enzymes used in Meju production [24].

Ethyl acetate, ethanol, propyl acetate, ethyl hexanoate, ethyl 2-hydroxypropanoate, and 1-octen-3-ol are found in alcoholic beverages, which are fermented by molds and yeasts. Twelve compounds (2-propanone, methyl acetate, 2-pentanone, isobutyl acetate, 2methyl-1-propanol, ethyl 2-methylbutyrate, 3-methyl-1-butyl acetate, 5-methyl-2-hexanone, 2-heptanone, 1-pentanol, 3-hydroxy-2-butanone, and 1-hexanol) have sweetish, fruity, banana, or buttery odors. In contrast, 2-methylpropanoic acid, 3-methyl-1-butanol, and benzaldehyde produce odors such as rancid butter, pungent, and bitter almond [25].

The content of 2-pentanone, isobutyl acetate, 3-hydroxy-2-butanone, 2-heptanone, 2,5-dimethylpyrazine, and benzaldehyde simultaneously increased after fermentation by B. velezensis (Figure 2). The odors of these compounds are sweet, such as banana, creamy, and almond. In contrast, ethyl acetate, ethanol, and 1-octen-3-ol were detected in Meju with high $A$. oryzae content. In particular, the content of 1 -octen-3-ol, which has a mushroom odor, decreased in Groups $C$ and D after fermentation with Bacillus starter. In addition, the content of 1-hexanol and 2-ethylhexanol, which have fresh grass and floral odors, was high in Group A and notably reduced in Groups C and D. Seo et al. reported that the contents of ethyl acetate in soybean koji fermented by Bacillus amyloliquefaciens or A. oryzae are $1.60 \pm 0.11$ and $2.49 \pm 0.13 \mathrm{mg} / \mathrm{kg}$, respectively. Moreover, koji fermented by $B$. amyloliquefaciens has a higher 2-methylpropanoic acid content $(2.81 \pm 0.15)$ than the koji fermented by $A$. oryzae (1.24 \pm 0.04$)$. In particular, ethyl 2-methylbutyrate (butanoate) is only detected in koji fermented with B. amyloliquefaciens (1.67 \pm 0.07$)$ [26]. Our results suggested that the Meju co-inoculated the Bacillus and fungi demonstrated improved flavor, whereas the one inoculated with only fungi did not.

Pent-1-en-3-ol, 4-ethenylpheol, 2-pheylethanol, and phenol were detected in samples fermented by B. velezensis, whereas 2-methylpropanal and 2-phenylbut-2-enal were detected in those fermented by A. oryzae. Moreover, benzaldehyde, 2-pentylfuran, hexanal, and 2-phenylacetaldehyde were detected in both. 
(a)

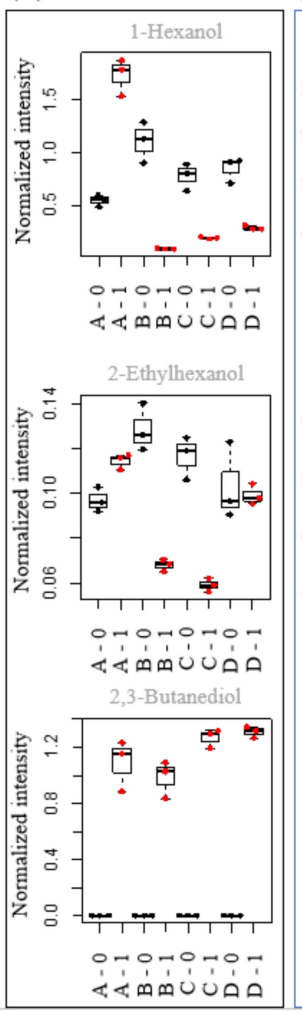

(b)

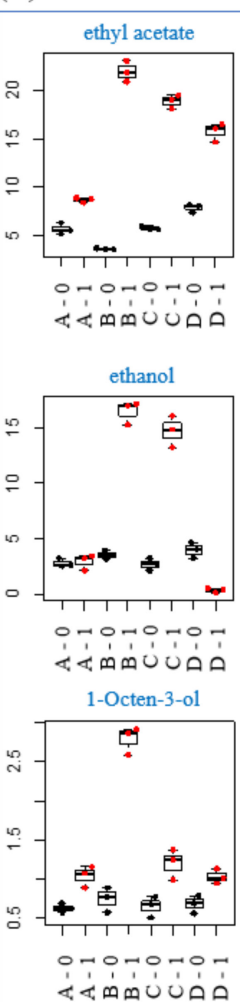

(c)
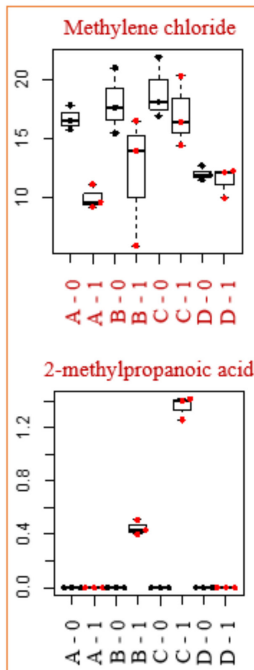

Ethyl 2-methylbutyrate

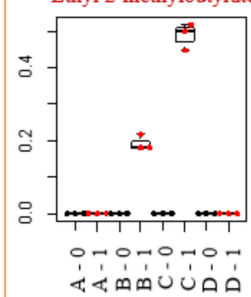

(d)
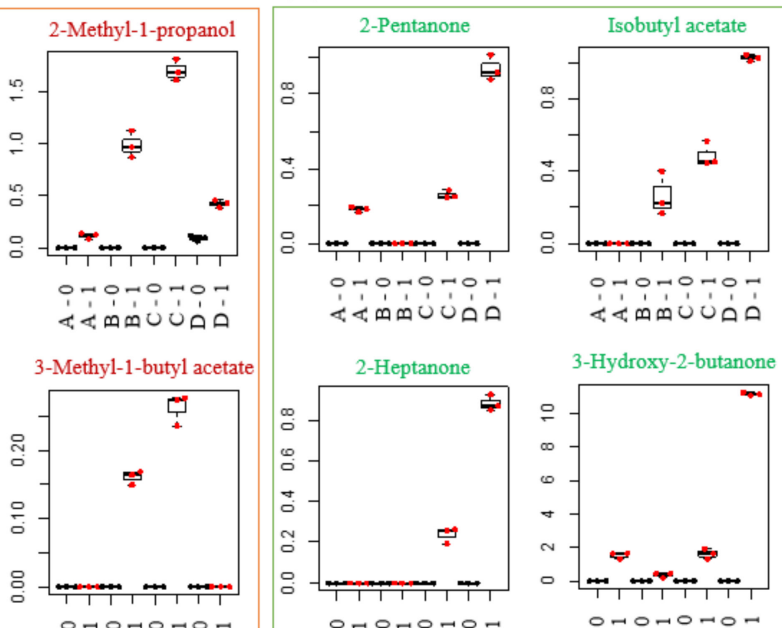

그응-

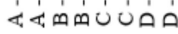

3-Methyl-1-butanol
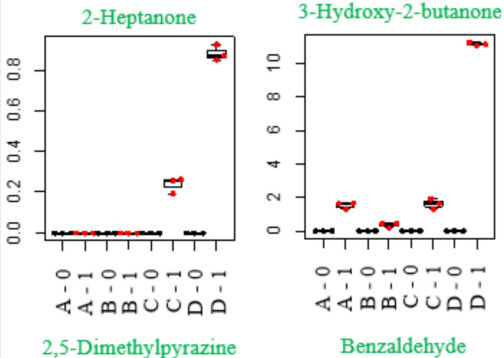

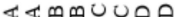

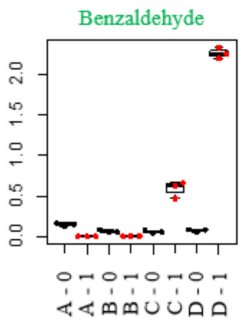

Figure 2. Boxplots derived from GC-MS data from Meju samples reveal metabolic changes during fermentation with different starters. (a) Volatiles increased in Group A as control without starter, (b) volatiles increased in Group B inoculated with A. oryzae KCTC 46471, (c) volatiles increased in Group $C$ inoculated individually with $B$. velezensis and $A$. oryzae, and (d) volatiles increased in Group D co-inoculated with $B$. velezensis and $A$. oryzae between pre- and post-fermentation for $0-1 \mathrm{~d}$.

\subsection{Comparison of Metabolite Profiles}

To evaluate the differences in primary metabolites among Meju samples, multi-factor analysis (MFA) and PLS-DA were performed using GC-TOF-MS or metagenome data in Figure 3. The MFA score plot showed 50.30\% and 26.26\% variances for F1 and F2, respectively. After fermentation, Groups B and C moved into quadrant 1, whereas Groups A and D moved into quadrants 3 and 4, respectively; each sample, fermented for one day, was separated in the MFA score plot, indicating that the Meju samples produced the metabolites, including fatty acids, free sugars, and sugar alcohols, owing to the inoculated starters. The quality parameters of the PLS-DA model were as follows: R2X $=0.796$, $\mathrm{R} 2 \mathrm{Y}=0.285$, and $\mathrm{Q} 2=0.137$ for bacteria and fermentation period metabolites. The values of the cross-validation ( $\mathrm{R} 2$ intercept $<0.2$ and Q2 intercept $<-0.1$ ) indicated the statistical acceptability of the PLS-DA models for bacterial and fungal metabolomic analysis, although the $p$-values in the PLS-DA score plots were higher than 0.05. Taken together, these results suggest that the metabolites of Meju during fermentation differ remarkably depending on the starter and inoculation method. 
(a)
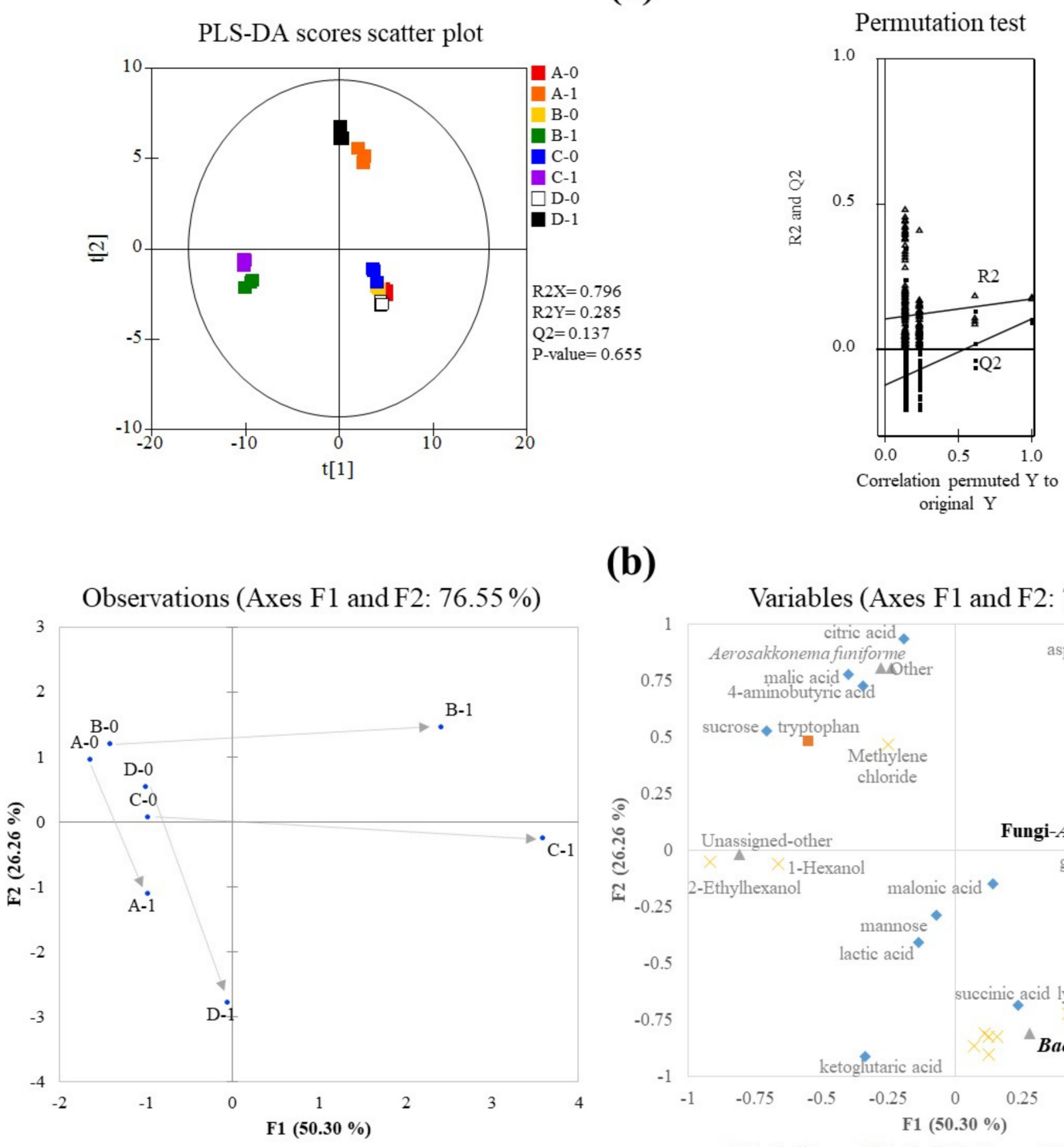

(b)

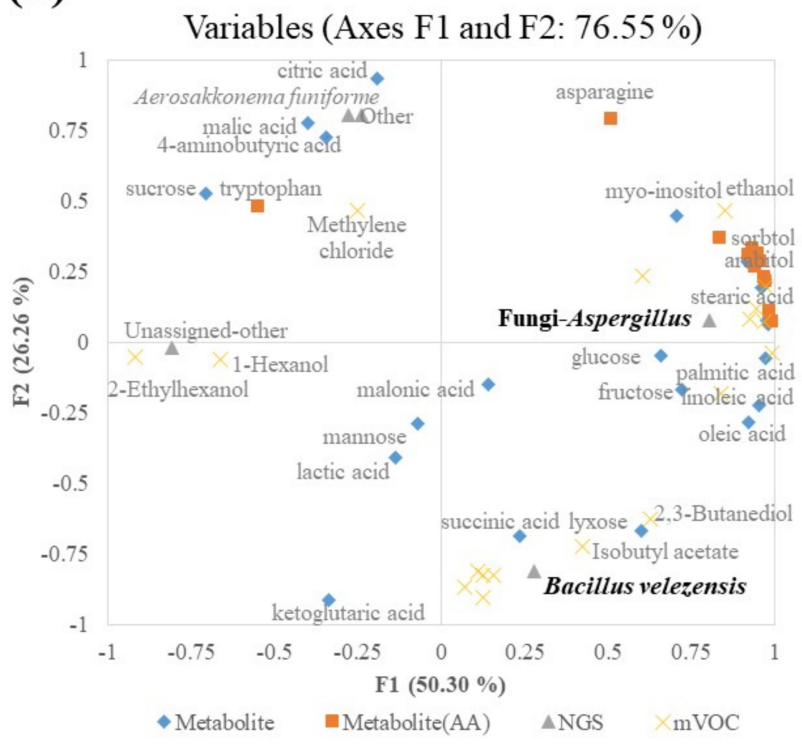

Figure 3. PLS-DA (a) was performed using GC-TOF-MS data and multi-factor analysis, (b) score plot from the set of GC-MS and NGS data from Meju samples reveal metabolic changes during fermentation among samples using different starters. Here, the datasets for raw substrates are indicated: $\diamond$, metabolites dataset except for amino acids; $\mathbf{\square}$, metabolites including only amino acids; $\times$, microbial volatile datasets; $\boldsymbol{\Lambda}$, metagenome datasets based on $16 \mathrm{~S}$ rRNA and ITS sequences.

\subsection{Heatmaps of Identified Metabolites}

Among the metabolites found by GC-MS, the statistical results of the normalized metabolites showed the metabolites changed significantly during Meju fermentation. Thirtythree Meju metabolites were identified (data not shown). In particular, metabolites, such as organic acids (e.g., lactic acid, malic acid, 4-aminobutyric acid, and ketoglutaric acid), sugars (e.g., sucrose), and amino acids (e.g., alanine, oxoproline, asparagine, and tryptophan), with variable importance in projection values $>1.0$ were identified as associated with energy metabolism. A. oryzae contributed more metabolites than B. velezensis.

Changes in the levels of metabolites identified in the four Meju groups were visualized according to metabolic pathways based on heatmaps (Figure 4). In Group B, the levels of metabolites, such as fructose, sorbitol, citric acid, and malic acid, were significantly higher than those in Groups C and D. 


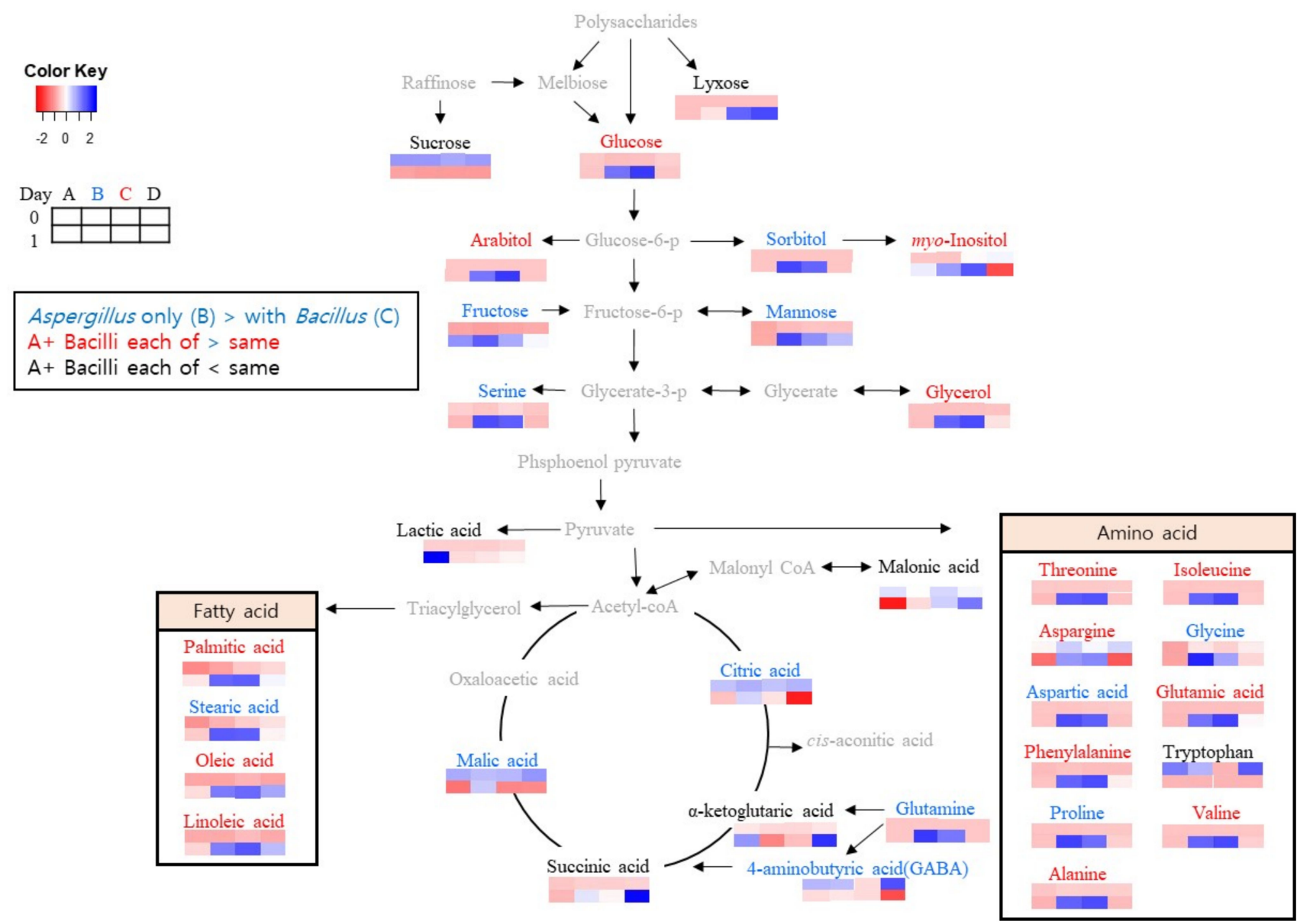

Figure 4. Scheme of a metabolic pathway based on the heatmap generated from GC-MS data from Meju samples reveals metabolic changes during fermentation for 0-24 h among samples using different starters. Metabolite compounds described as color of compound name: Blue, higher levels in Group B inoculated only A. oryzae than in Group C individually inoculated with A. oryzae and $B$. velezensis; Red, higher levels in Group C than in Group D co-inoculated simultaneously with A. oryzae and B. velezensis; Black, higher levels in Group D than in Group C.

Meju in Group C contained metabolites of glucose, myo-inositol, glycerol, and fatty acids (palmitic, stearic, oleic, and linoleic acids), which were more highly detected in this other group than in the other groups. Of these metabolites, malonic acid and lactic acid were produced by Bacillus, supporting findings described by Seo et al. [26]. According to Kang, the tryptophan, lyxose, succinic acid, $\alpha$-ketoglutaric acid, and 4-aminobutyric acid (GABA) content in soybeans $(16.93+0.10)$ is higher than that in Meju and Doenjang (9.30 $\mathrm{mg} / 100 \mathrm{~g}$ and $7.53 \mathrm{mg} / 100 \mathrm{~g}$ (dry basis), respectively) [27].

A correlation analysis conducted by Lee et al. showed that $B$. velezensis is linked to the production of fatty acids [28]. These results follow those of our study. The composition of Group C was $94 \%$ B. velezensis (Figure 1), and the production of fatty acids, such as palmitic acid, oleic acid, and linoleic acid, was higher than that in the other groups (Figure 3).

\subsection{Predictive Functional Genes in Fungal and Bacterial Inoculants of Meju}

The PICRUSt analytical tool is used in metagenomics and allows for inference of the functional profile of a microbial community based on a marker gene survey among samples [24]. In this study, to predict the genetic amino acid pathway from microorganisms, the Meju samples produced using $A$. oryzae inoculation with or without $B$. velezensis were aggregated into two clusters: pre- and post-fermentation (Figure 5). 


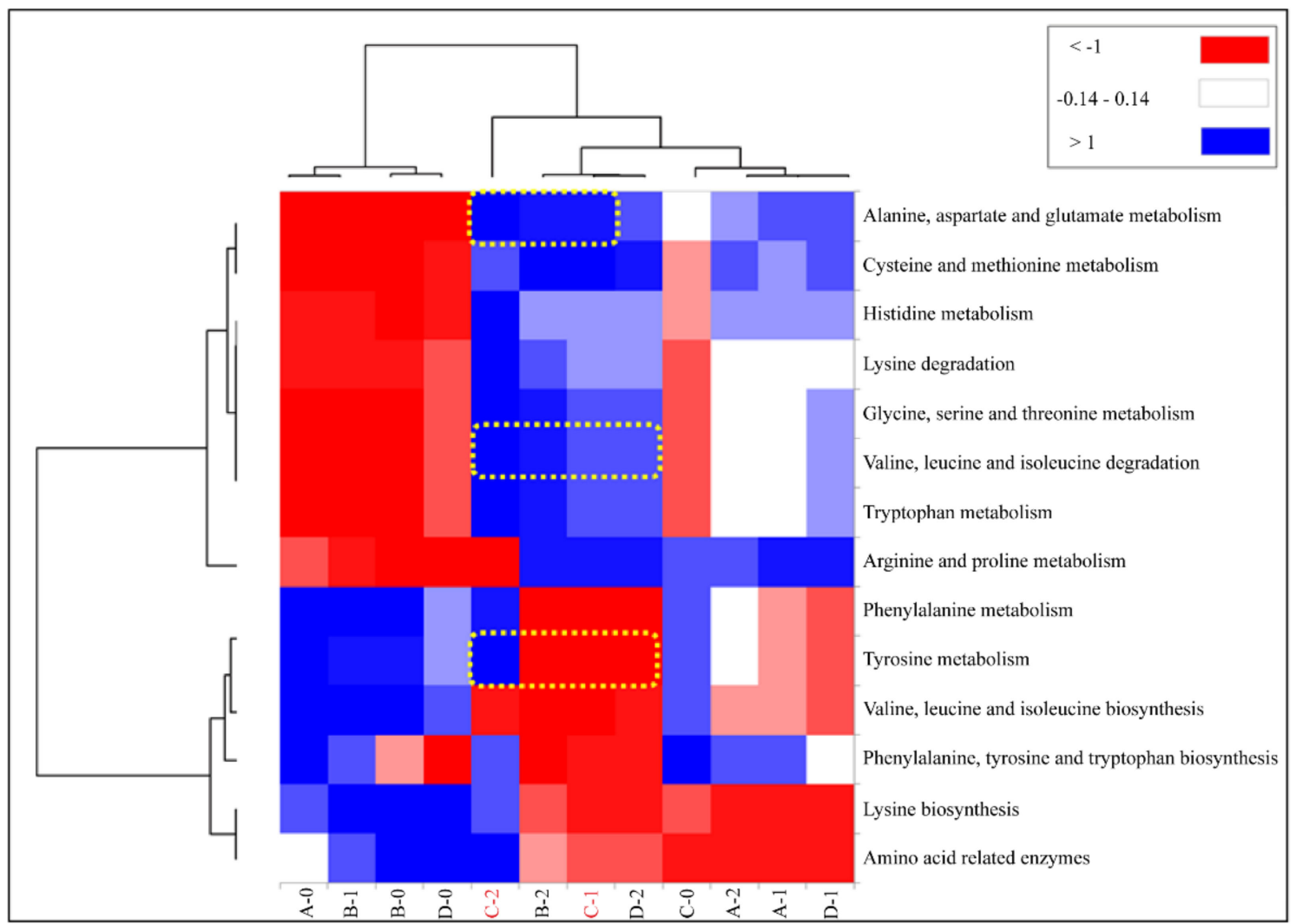

Figure 5. PICRUSt analysis of the operational taxonomic unit data of the metagenome reveals metabolic changes in fermented Meju using different starters. The experimental groups were inoculated as follows: A, control without starter; B, Aspergillus oryzae single inoculation group; C, Bacillus velezensis and $A$. oryzae individual inoculation group; and $\mathrm{D}, B$. velezensis and $A$. oryzae co-inoculation group, during fermentation for $0-2 \mathrm{~d}$.

For the initial fermentative samples, red clusters were related to the metabolism of alanine, aspartate, glutamate, cysteine, methionine, histidine, glycine, serine, threonine, and tryptophan and the degradation of lysine, valine, leucine, isoleucine, and tryptophan. Blue clusters were related to the metabolism of phenylalanine and tyrosine and biosynthesis of valine, leucine, isoleucine, phenylalanine, tyrosine, lysine, and amino acid-related enzymes for pre-fermentation. The difference between the clusters indicates there are more degradation function genes grouped in the red cluster and more biosynthesis function genes grouped in the blue cluster. The samples showed changes in their functional profiles at $24-48 \mathrm{~h}$ of fermentation compared with those at $0 \mathrm{~h}$. Sample $\mathrm{C}$ showed a distinct amino acid profile among the starter-inoculated groups; at $0 \mathrm{~h}$, it was classified into the same cluster as samples B and D at $24 \mathrm{~h}$ were, demonstrating that the fermentation rate of sample $C$ was faster than the other samples. After $24 \mathrm{~h}$, sample $\mathrm{C}$ showed metabolism of lysine, which is essential for nutrition, which moved it to the blue cluster. These results indicate that the inoculation method for sample $\mathrm{C}$ can predict the amino acid pathway from microbial communities. We propose that differences in inoculation determine the quality characteristics of Meju.

Microbial communities in fermented foods have been reported to have sensory characteristics such as those of Korean soybean paste. The amino acids degraded by bacteria are crucial factors that can determine the taste quality of soybean paste. Amino acids are responsible for sweet (lysine, alanine, glycine, serine, and threonine), umami (glutamate and aspartate), and bitter (isoleucine and phenylalanine) tastes [29-32]. Furthermore, this study showed that bacterial communities in $M e j u$ are responsible for the production of 
amino acids. Therefore, we focused on the function of bacteria in the predicted amino acid metabolism in Meju.

\section{Conclusions}

Meju is used in the production of soybean paste (Doenjang), soy sauce (Ganjang), and red pepper paste (Gochujang). The genera Aspergillus and Bacillus found in Meju during fermentation to influence its characteristic metabolites, taste, and aroma. However, $B$. velezensis, which is the most predominantly detected species in Korean soybean sauces, affects the growth of useful $A$. oryzae. Therefore, we investigated the differences in properties and metabolites in Meju samples fermented by Aspergillus oryzae alone or by Bacillus velezensis. We demonstrated that fermented Meju, with an amino-type nitrogen content of $776 \mathrm{mg} \%$, can be manufactured using the fungi and bacteria separately (C group) over 1 day. Notably, the Meju produced from $A$. oryzae and B. velezensis using the inoculation method showed favorable metabolites, such as glucose, myo-inositol, glycerol, and fatty acids (palmitic, stearic, oleic, and linoleic acids), compared with Meju fermented by A. oryzae alone.

Consequently, we propose that the inoculation method using both fungal and bacterial starters can be effectively used in manufacturing Meju and contribute to fungal survival and fermentation.

Author Contributions: Conceptualization, S.-Y.K.; methodology, N.-Y.G.; validation, S.-Y.K.; formal analysis, W.-S.J.; investigation, H.-M.G.; writing—original draft preparation, N.-Y.G.; writing—review and editing, Y.-J.J.; visualization, S.-Y.K.; supervision, S.-Y.K.; project administration, S.-H.Y. All authors have read and agreed to the published version of the manuscript.

Funding: This research was funded by the Research Program for Agricultural Science \& Technology Development, grant number PJ013833 and the National Institute of Agricultural Sciences, Rural Development Administration, Republic of Korea (Project No. PJ013833).

Institutional Review Board Statement: Not applicable.

Informed Consent Statement: Not applicable.

Data Availability Statement: Data is contained within the article.

Conflicts of Interest: The authors declare no conflict of interest. The funders had no role in the design of the study; in the collection, analyses, or interpretation of data; in the writing of the manuscript; or in the decision to publish the results.

\section{References}

1. Gil, N.Y.; Song, J.; Eom, J.S.; Park, S.Y.; Choi, H.S. Changes of physicochemical properties of Cheonggukjang prepared with various soybean cultivars and Bacillus subtilis HJ18-9. Korean J. Food Preserv. 2016, 23, 811-818. [CrossRef]

2. Cho, K.M.; Lim, H.J.; Kim, M.-S.; Kim, D.S.; Hwang, C.E.; Nam, S.H.; Joo, O.S.; Lee, B.W.; Kim, J.K.; Shin, E.C. Time course effects of fermentation on fatty acid and volatile compound profiles of Cheonggukjang using new soybean cultivars. J. Food Drug Anal. 2017, 25, 637-653. [CrossRef] [PubMed]

3. Omoni, A.O.; Aluko, R.E. Soybean foods and their benefits: Potential mechanisms of action. Nutr. Rev. 2005, 63, 272-283. [CrossRef] [PubMed]

4. Gil, N.Y.; Choi, B.Y.; Park, S.Y.; Cho, Y.S.; Kim, S.Y. Physicochemical properties of Doenjang using grain type Meju fermented by Aspergillus oryzae and protease. Korean J. Food Preserv. 2017, 24, 697-706. [CrossRef]

5. Lee, S.S.; Park, K.H.; Choi, K.J.; Won, S. Identification and isolation of zygomycetous fungi found on Maeju, a raw material of Korean traditional soysources. Kor. J. Mycol. 1993, 21, 172-187. (In Korean)

6. Jung, J.Y.; Lee, S.H.; Jeon, C.O. Microbial community dynamics during fermentation of doenjang-meju, traditional Korean fermented soybean. Int. J. Food Microbiol. 2014, 185, 112-120. [CrossRef]

7. Jo, H.D.; Lee, H.A.; Jeong, S.J.; Kim, J.H. Purification and characterization of a major fibrinolytic enzyme from Bacillus amyloliquefaciens MJ5-41 isolated from Meju. J. Microbiol. Biotechnol. 2011, 21, 1166-1173. [CrossRef] [PubMed]

8. Kim, Y.S.; Jeong, J.O.; Cho, S.H.; Jeong, D.Y.; Uhm, T.B. Antimicrobial and biogenic amine-degrading activity of Bacillus licheniformis SCK B11 isolated from traditionally fermented red pepper paste. Korean J. Microbiol. 2012, 48, 163-170. [CrossRef]

9. Stein, T. Bacillus subtilis antibiotics: Structures, syntheses and specific functions. Mol. Microbiol. 2005, 56, 845-857. [CrossRef]

10. Ministry of Food and Drug Safety (MFD). Specifications and Standards for Foods; No. 2019-81; MFDS: Osong, Korea, 2019. 
11. Borriss, R.; Chen, X.H.; Rückert, C.; Blom, J.; Becker, A.; Baumgarth, B.; Fan, B.; Pukall, R.; Schumann, P.; Spröer, C.; et al. Relationship of Bacillus amyloliquefaciens clades associated with strains DSM 7T and FZB42T: A proposal for Bacillus amyloliquefaciens subsp. amyloliquefaciens subsp. nov. and Bacillus amyloliquefaciens subsp. plantarum subsp. nov. based on complete genome sequence comparisons. Int. J. Syst. Evol. Microbiol. 2011, 61, 1786-1801.

12. Dunlap, C.A.; Kim, S.J.; Kwon, S.W.; Rooney, A.P. Bacillus velezensis is not a later heterotypic synonym of Bacillus amyloliquefaciens; Bacillus methylotrophicus, Bacillus amyloliquefaciens subsp. plantarum and 'Bacillus oryzicola' are later heterotypic synonyms of Bacillus velezensis based on phylogenomics. Int. J. Syst. Evol. Microbiol. 2016, 66, 1212-1217.

13. Kim, E.Y.; Kim, D.G.; Kim, Y.R.; Choi, S.Y.; Kong, I.S. Isolation and identification of halotolerant Bacillus sp. SJ-10 and characterization of its extracellular protease. Misainmurhag Hoiji. 2009, 45, 193-199. (In Korean)

14. Chang, M.; Moon, S.H.; Chang, H.C. Isolation of Bacillus velezensis SSH100-10 with antifungal activity from Korean traditional soysauce and characterization of its antifungal compounds. Korean J. Food Preserv. 2012, 19, 757-766. [CrossRef]

15. Moon, S.H.; Park, Y.J.; Kim, I.C.; Chang, H.H. Isolation and characterization of Bacillus velezensis SS360-1 from seed soy sauce. Korean J. Community Living Sci. 2018, 29, 49-58. [CrossRef]

16. Kim, S.Y.; Park, S.Y.; Cho, Y.S.; Gil, N.Y.; Choi, B.Y. Bacillus amyloliquefaciens NY12-2 Strain Having Antibiotic Activity against Bacillus cereus and Inhibitory Activity on Biogenic Amine and Compositions Thereof. Korean Patent No. 10-2000475, 10 July 2019.

17. Association of Official Analytical Chemists (A.O.A.C.). Official Methods of Analysis, 15th ed.; A.O.A.C.: Washington, DC, USA, 1990.

18. Langille, M.G.; Zaneveld, J.; Caporaso, J.G.; McDonald, D.; Knights, D.; Reyes, J.A.; Clemente, J.C.; Burkepile, D.E.; Vega Thurber, R.L.; Knight, R.; et al. Predictive functional profiling of microbial communities using 16S rRNA marker gene sequences. Nat. Biotechnol. 2013, 9, 814-821. [CrossRef] [PubMed]

19. Mahdi, M.A. Isolation four species of Bacillus and tested activity against some filamentous fungi. Eur. J. Exp. Biol. 2013, 3 , 495-498.

20. Choi, J.; Kim, M.H.; Shon, M.Y.; Park, S.K.; Choi, S.-D.; Hong, U. Production and quality properties of capsule type Meju prepared with Rhizopus oligosporus. Korean J Food. Preserv. 2002, 9, 315-320. (In Korean)

21. Liu, Y.; Teng, K.; Wang, T.; Dong, E.; Zhang, M.; Tao, Y.; Zhong, J. Antimicrobial Bacillus velezensis HC6: Production of three kinds of lipopeptides and biocontrol potential in maize. J. Appl. Microbiol. 2020, 128, 242-254. [CrossRef]

22. Thu, N.K.; Tanabe, Y.; Yoshida, M.; Matsuura, H.; Watanabe, M.M. Aerosakkonema funiforme gen. et sp. nov. (Oscillatoriales), a new gas-vacuolated oscillatorioid cyanobacterium isolated from a mesotrophic reservoir. Phycologia 2012, 51, 672-683. [CrossRef]

23. Lee, L.; Heo, S.; Jeong, D.W. Fungal microbial community profiles of meju, solar salt, and doenjang using pyrosequencing. Microbiol. Biotechnol. Lett. 2019, 47, 354-358. [CrossRef]

24. Park, J.S.; Lee, M.Y.; Kim, K.S.; Lee, T.S. Volatile flavor components of soybean paste (doenjang) prepared from different types of strains. Korean J. Food Sci. Technol. 1994, 26, 255-260.

25. Kim, S.; Chen, J.; Cheng, T.; Gindulyte, A.; He, J.; He, S.; Li, Q.; Shoemaker, B.A.; Thiessen, P.A.; Yu, B.; et al. PubChem in 2021: New data content and improved web interfaces. Nucleic Acids Res. 2019, 49, 1388-1395. [CrossRef]

26. Seo, H.S.; Lee, S.; Singh, D.; Shin, H.W.; Cho, S.A.; Lee, C.H. Untargeted metabolite profiling for koji-fermentative bioprocess unravels the effects of varying substrate types and microbial inocula. Food Chem. 2018, 266, 161-169. [CrossRef] [PubMed]

27. Kang, S.H. Physicochemical and Functional Characteristics of Traditional Meju and Doenjang with Soybean in Different Seeding Times. Ph.D. Thesis, Seoul National University, Seoul, Korea, 2012.

28. Lee, S.; Lee, S.; Singh, D.; Oh, J.Y.; Jeon, E.J.; Ryu, H.S.; Lee, D.W.; Kim, B.S.; Lee, C.H. Comparative evaluation of microbial diversity and metabolite profiles in doenjang, a fermented soybean paste, during the two different industrial manufacturing processes. Food Chem. 2017, 221, 1578-1586. [CrossRef] [PubMed]

29. Namgung, H.J.; Park, H.J.; Cho, I.H.; Choi, H.K.; Kwon, D.Y.; Shim, S.-M.; Kim, Y.S. Metabolite profiling of doenjang, fermented soybean paste, during fermentation. J. Sci. Food Agric. 2010, 90, 1926-1935. [CrossRef] [PubMed]

30. Kim, M.J.; Kwak, H.S.; Jung, H.Y.; Kim, S.S. Microbial communities related to sensory attributes in Korean fermented soy bean paste (doenjang). Food Res. Int. 2016, 89 Pt 1, 724-732. [CrossRef] [PubMed]

31. Jung, W.Y.; Jung, J.Y.; Lee, H.J.; Jeon, C.O. Functional characterization of bacterial communities responsible for fermentation of Doenjang: A traditional Korean fermented soybean paste. Front. Microbiol. 2016, 7, 827. [CrossRef] [PubMed]

32. Yue, X.; Li, M.; Liu, Y.; Zhang, X.; Zheng, Y. Microbial diversity and function of soybean paste in East Asia: What we know and what we don't. Curr. Opin. Food Sci. 2021, 37, 145-152. [CrossRef] 